\title{
Confusing Difficulty to Breath
}

\author{
Genevieve B. Payettea, c , Maxime Lamarre-Cliche ${ }^{\mathrm{b}}$, \\ Mikhael Laskine ${ }^{\mathrm{b}}$
}

\begin{abstract}
Hereby presented is a case of a rather unusual community-acquired Mycoplasma pneumonia (M. pneumonia) infection in a 47 year-old woman with pneumonia associated with confusion, sleepiness and disorganisation before her hospital admission. Later on, she developed a clinically significant cold agglutinin hemolytic anemia which required a blood transfusion support. Other extrapulmonary symptoms should be looked for in patients infected with M. pneumonia. The central nervous system (CNS) involvement being the most common one, other systems such as cardiac, renal, skin, gastro-intestinal and rheumatologic could be affected. M. pneumonia should be searched for by serology, if available in your hospital, as it is one of the common atypical pneumonia that needs to be kept in mind by all primary care physicians. Recognizing this infectious agent is crucial for everybody as the patient needs to be isolated as it is an airborne transmission and they need to stay in a warm room to prevent or reduce the associated hemolytic anemia complication. It also typically responds to macrolides and fluoroquinolones specifically but doxycycline can also be used in the treatment arsenal.
\end{abstract}

Keywords: Mycoplasma pneumonia; Pneumonia; Confusion; Hemolytic anemia; Cold agglutinins

\section{Introduction}

With the high prevalence of community-acquired pneumonia, especially during the winter season, the review of this interesting and unusual case of a young adult woman with

\footnotetext{
Manuscript accepted for publication May 22, 2013

anternal Medicine Resident, University Hospital of Montreal (CHUM), 3840 St-Urbain, Montreal, Quebec, H2W 1T8, Canada

${ }^{\mathrm{b}}$ Internal Medicine, University Hospital of Montreal (CHUM), 3840

St-Urbain, Montreal, Quebec, H2W 1T8, Canada

${ }^{\mathrm{c}}$ Corresponding author: Genevieve B. Payette, University Hospital of

Montreal (CHUM), 3840 St-Urbain, Montreal, Quebec, H2W 1T8,

Canada. Email: genevieve.payette@gmail.com

doi: http://dx.doi.org/10.4021/jmc1233w
}

infectious pulmonary symptoms, central nervous system involvement and hemolytic anemia is presented.

\section{Case Report}

A 47 year-old female presents herself at the emergency department with fatigue, cough and an involuntary weight loss. She had consulted in another hospital prior to her visit where she was diagnosed with a community-acquired pneumonia and was sent home with amoxicillin / clavulanate for a total of 14 days. However, she still felt ill and she wanted a second opinion.

Ms. $\mathrm{X}$ is not known for any significant disease and had only had an acute pyelonephritis and a bronchitis in the past. She is also a smoker with 15 packs-year. She had undergone an ovariectomy. She was born in the Democratic Republic of the Congo and had lived a few years in Belgium before moving to Canada.

Upon her arrival, her vital signs are stable, apart from a tachycardia of 114 beats per minute and a saturation of $95 \%$ with ambient air. She has a non-productive cough and crepitants could be heard at the base of the left lung.

It is noted that she has few signs of CNS involvement: sleepiness, disorganisation and disorientation. She had also visual hallucinations and possibly a paranoid delirium before coming to the emergency according to her son.

She is found to be in an inflammatory state with her sedimentation rate at $137 \mathrm{~mm} / \mathrm{H}$ (upper limit of normal range 20 $\mathrm{mm} / \mathrm{H}$ ), her C-reactive protein at $21 \mathrm{mg} / \mathrm{L}$ (upper limit of normal range $5 \mathrm{mg} / \mathrm{L}$ ) and her ferritin at $1886 \mathrm{ug} / \mathrm{L}$ (upper limit of normal range $90 \mathrm{ug} / \mathrm{L}$ for her age and sex). Ms. X's white blood cells are at $21.2 \times 10^{9} / \mathrm{L}$ (upper limit of normal range $11 \times 10^{9} / \mathrm{L}$ ) with neutrophils at $14.4 \times 10^{9} / \mathrm{L}$ (upper limit of normal range $7.7 \times 10^{9} / \mathrm{L}$ ) and her platelets at $656 \times$ $10^{9} / \mathrm{L}$ (maximum of 743 - upper limit of normal range 450 $\left.\times 10^{9} / \mathrm{L}\right)$.

On the pulmonary X-ray, we can see a bilateral pattern of reticulo-nodularity (Fig. 1). Pneumonia is diagnosed and she is switched to daily levofloxacin 500 milligrams.

A normocytic normochromic anemia is discovered with a hemoglobin level of $78 \mathrm{~g} / \mathrm{L}$, rapidly progressing to $61 \mathrm{~g} / \mathrm{L}$ 


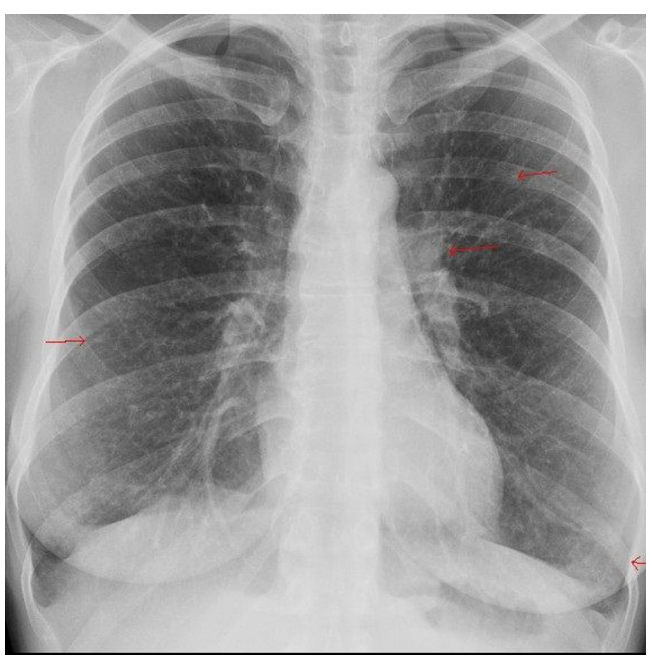

Figure 1. Ms X's lung X-ray showing the bilateral pattern of reticulo-nodularity.

with a normal iron profile, as her TSH, B12 vitamin and folic acid. A haemolytic anemia is diagnosed with $\mathrm{LDH}$ at 720 $\mathrm{U} / \mathrm{L}$ (upper limit of normal range $205 \mathrm{U} / \mathrm{L}$ ) and haptoglobin less than $0.07 \mathrm{~g} / \mathrm{L}$ (lower limit of normal range $0.35 \mathrm{~g} / \mathrm{L}$ ). This anemia is found to be reactive with cold agglutinins anti-I IgM.

Two blood transfusions and a heated room were needed to stabilise the patient. 2.

We can see cold agglutinins hemolytic anemia in Figure

Within the differential diagnosis of the clinical picture we eliminated HIV (negative antibody and antigen), syphilis (negative EIA), systemic erythematous lupus (negative antinuclear antibody, normal complement 3 and 4), tuberculosis (negative serology and 4 negative Zielh) and mononucleosis (negative monotest). She underwent a full work-up for neoplastic or autoimmune disease (cerebral, thoracic and abdominal scans and a mammography) which came back negative. Finally, the M. pneumonia serology came back positive with both $\operatorname{IgG}$ at 8.91 (upper limit of normal range 1.10 ) and IgM at 8.57 (same upper limit as IgG) in this clinical setting of pneumonia with a cold agglutinin haemolytic anemia and CNS involvement. The pulmonary X-ray 8 days post-admission showed an almost resolution of the reticulonodular pattern.

\section{Discussion}

M. pneumonia, hominis and genitalium are the three species of Mycoplasma mostly involved in human infections. They are short rods that cannot be Gram stained (they do not have a cell wall) [1]. They can grow both in aerobic and anaerobic media. Mycoplasma belongs to the class of Mollicutes, along with Ureaplasma.

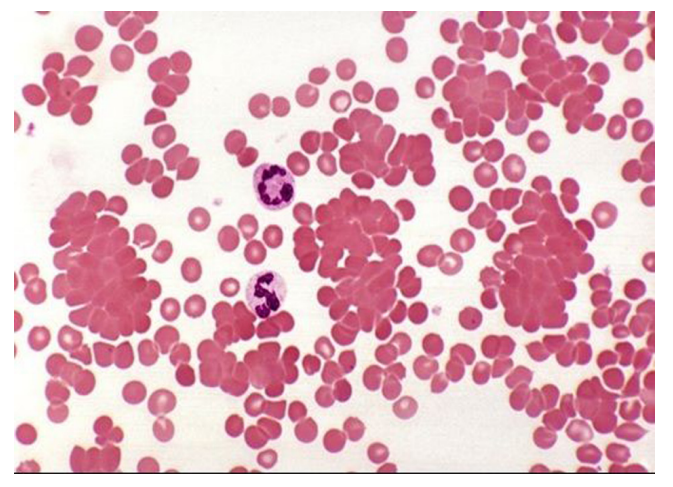

Figure 2. Cold agglutinins hemolytic anemia. Courtesy of Professor Kristine Krafts, md, University of Minnesota School of Medicine.

M. pneumonia is one of the common atypical germs involved in community-acquired pneumonia. It usually affects children or military recruits, but can also be found in adults [2]. Clinically, it can vary from almost no symptoms, upper respiratory tract symptoms only, to pneumonia with or without extrapulmonary symptoms, and even empyema.

The involvement of extrapulmonary organs can occur not only by autoimmunity, but also by direct invasion [1]. Of the extrapulmonary symptoms, the neurological ones are the most frequent with encephalitis [3]. Other organ implications can be hematologic (IgM cold agglutinins) through autoantibodies against M. pneumonia's glycolipid antigens [4]. This cold agglutinin hemolytic anemia is also rarely clinically significant, which makes our patient's case even more unusual as she had her hemoglobin level dropped down to 61 $\mathrm{g} / \mathrm{L}$ and necessitated blood transfusions.

Otherwise, the cardio-vascular system can be affected with symptoms of pericarditis and arrhythmia. The renal (glomerulonephritis) and gastrointestinal (hepatitis, pancreatitis) systems may also be affected. Rheumatologic (arthritis, Raynaud) and skin (rash) involvement are possible as well [2]. Hence, other clinical and para-clinical investigations would implicate an electrocardiogram, a urine analysis, liver and pancreatic enzymes and a careful exam of skin and joints, so as to eliminate synovitis or an associated rash.

Because transmission of $M$. pneumonia is by airborne droplets and as of the potential hematologic complication, patients infected with M. pneumonia need to be isolated in a warm room. Treatment is often empirically started with a macrolide (azithromycin, clarithromycin or erythromycin) or a fluoroquinolone (levofloxacin or moxifloxacin) or a tetracycline derivative (doxycycline) for a total of 7 to 14 days $[1,5]$. Resistance in North America to these antibiotics is low.

\section{Summary}

When a patient presents infectious pulmonary symptoms and 
central nervous system involvement, it is important to look for Mycoplasma pneumonia with a serologic test. If suspected, treatment involves macrolides, fluoroquinolones or doxycycline. A respiratory isolation in a warm room is necessary in order to prevent the spread of the disease and the apparition or complications of cold-agglutinins hemolytic anemia.

\section{References}

1. Atkinson TP, Balish MF, Waites KB. Epidemiology, clinical manifestations, pathogenesis and laboratory detection of Mycoplasma pneumoniae infections. FEMS Microbiol Rev. 2008;32(6):956-973.
2. Kashyap S, Sarkar M. Mycoplasma pneumonia: Clinical features and management. Lung India. 2010;27(2):7585.

3. Waites KB, Balish MF, Atkinson TP. New insights into the pathogenesis and detection of Mycoplasma pneumoniae infections. Future Microbiol. 2008;3(6):635648.

4. Khan FY, M Ay. Mycoplasma pneumoniae associated with severe autoimmune hemolytic anemia: case report and literature review. Braz J Infect Dis. 2009;13(1):77-79.

5. Goto H. Multicenter surveillance of adult atypical pneumonia in Japan: its clinical features, and efficacy and safety of clarithromycin. J Infect Chemother. 2011;17(1):97-104. 